\title{
Peritonite em pacientes em Diálise Peritoneal Automatizada: 18 anos de experiência de um centro de diálise do sul do Brasil
}

\author{
Peritonitis in patients on Automated Peritoneal Dialysis: 18 years of experience in a \\ single dialysis center of southern Brazil
}
Peritonitis en pacientes en diálisis peritoneal automatizada: 18 años de experiencia en un centro de diálisis en el sur de Brasil

Amauri Braga Simonetti ${ }^{1 *}$, Sinara Guzzo Chioquetta ${ }^{2}$, Jairo José Caovilla ${ }^{1,2}$.

\begin{abstract}
RESUMO
Objetivo: Avaliar as taxas e os desfechos de peritonite, além de características gerais de pacientes submetidos à diálise peritoneal automatizada. Métodos: Trata-se de um estudo retrospectivo descritivo onde foram avaliados dados sociodemográficos, clínicos e laboratoriais de pacientes de um centro de diálise de Passo Fundo, RS, Brasil. Resultados: De 213 pacientes analisados, 88 apresentaram 181 episódios de peritonite. A idade média foi de 51,7 anos, $53,4 \%$ eram do sexo feminino e $36 \%$ eram diabéticos. Bactérias Gram-positivas foram a causa mais frequente $(37,7 \%)$, com predominância de Staphylococcus coagulasenegativos (SCN) em 14,7\% e Staphylococcus aureus em 13\% dos casos. Em 32\% dos episódios não houve detecção de micro-organismos. A taxa global de peritonite foi de 0,33 episódio/paciente/ano (ep./ano) e por micro-organismo foi de 0,048 para SCN, 0,042 para S. aureus e de 0,057 para bactérias Gram-negativas. Quanto aos desfechos, houve maior percentual de cura em peritonites por bactérias Gram-positivas (82\%) do que por Gram-negativas (60,6\%), infecções mistas $(50 \%)$ e fungos (18\%). Remoção de cateter e óbito foram mais frequentes nos casos de peritonite fúngica, polimicrobianas e por Gram-negativos. Conclusão: As taxas de peritonite encontram-se dentro dos limites preconizados pelas diretrizes internacionais.
\end{abstract}

Palavras-chave: Diálise Peritoneal, Peritonite, Insuficiência Renal Crônica.

\section{ABSTRACT}

Objective: To evaluate peritonitis rates and outcomes, as well as general characteristics of patients undergoing automated peritoneal dialysis. Methods: This is a descriptive retrospective study that evaluated sociodemographic, clinical and laboratory data of patients from a dialysis center in Passo Fundo, RS, Brazil. Results: Of 213 patients analyzed, 88 presented 181 episodes of peritonitis. The average age estimated was 51.7 years, $53.4 \%$ were female and $36 \%$ were diabetic. Gram-positive bacteria were the most frequent cause $(37.7 \%$ ), with a predominance of coagulase-negative Staphylococcus (CNS) in $14.7 \%$ and Staphylococcus aureus in $13 \%$ of cases. In $32 \%$ of the episodes there was no detection of microorganisms. The overall peritonitis rate was 0.33 episodes/patient/year (ep./year) and per microorganism was 0.048 for SCN, 0.042 for S. aureus and 0.057 for Gram-negative bacteria. Regarding outcomes, there was a higher percentage of cure in peritonitis by Gram-positive bacteria (82\%) than by Gram-negative bacteria (60.6\%), mixed infections (50\%) and fungi (18\%). Catheter removal and death were more frequent in cases of fungal, polymicrobial and gram-negative peritonitis. Conclusion: Peritonitis rates are within the limits recommended by international guidelines.

Keywords: Peritoneal Dialysis, Peritonitis, Chronic Renal Failure.

\footnotetext{
1Universidade Federal da Fronteira Sul (UFFS), Passo Fundo-RS.

*E-mail: amauri.simonetti@uffs.edu.br

${ }^{2}$ Hospital de Clínicas de Passo Fundo, Passo Fundo-RS.
}

SUBMETIDO EM: 11/2019

ACEITO EM: $12 / 2019$

PUBLICADO EM: 2/2020 


\section{RESUMEN}

Objetivo: El objetivo de este estudio fue evaluar las tasas y resultados de peritonitis, así como las características generales de los pacientes sometidos a diálisis peritoneal automatizada. Métodos: Este es un estudio descriptivo retrospectivo que evaluó datos sociodemográficos, clínicos y de laboratorio de pacientes de un centro de diálisis en Passo Fundo, RS, Brasil. Resultados: De 213 pacientes analizados, 88 tuvieron 181 episodios de peritonitis. La edad promedio fue de 51.7 años, el $53.4 \%$ eran mujeres y el $36 \%$ eran diabéticos. Las bacterias Gram positivas fueron la causa más frecuente (37.7\%), con predominio de Staphylococcus coagulasa negativo (SCN) en 14.7\% y Staphylococcus aureus en $13 \%$ de los casos. En el $32 \%$ de los episodios no hubo detección de microorganismos. La tasa general de peritonitis fue de 0,33 episodios / paciente / año (ep./año) y por microorganismo fue de 0,048 para SCN, 0,042 para S. aureus y 0,057 para bacterias Gram negativas. En cuanto a los resultados, hubo un mayor porcentaje de cura en la peritonitis por bacterias Gram positivas (82\%) que por bacterias Gram negativas (60.6\%), infecciones mixtas $(50 \%)$ y hongos (18\%). La remoción del catéter y la muerte fueron más frecuentes en casos de peritonitis micótica, polimicrobiana y Gram negativa. Conclusión: Las tasas de peritonitis están dentro de los límites recomendados por las directrizes internacionales.

Palabras clave: Diálisis Peritoneal, Peritonitis, Insuficiencia Renal Crónica.

\section{INTRODUÇÃO}

A doença renal crônica (DRC) é um problema de saúde pública no Brasil e no mundo, sendo Diabetes Melito (DM) e Hipertensão Arterial Sistêmica (HAS) as principais causas na população adulta (BROWN MC, et al.; SESSO RCC, et al., 2017; PICCOLLI AP, et al., 2017). A DRC em estágio terminal tem como tratamento a Terapia Renal Substitutiva (TRS), que inclui hemodiálise, diálise peritoneal (DP) e transplante renal. A hemodiálise é realizada três vezes por semana e utiliza um equipamento específico que filtra o sangue diretamente e o devolve ao corpo do paciente com menos impurezas, sendo realizada em centros especializados. A DP usa um equipamento que infunde e drena uma solução especial diretamente no abdômen do paciente, sem contato direto com o sangue, sendo realizada diariamente na casa do paciente ou em hospital (DURVASULA RV e HIMMERFALB, 2011).

Em particular, na DP tem-se como modalidade de tratamento a Diálise Peritoneal Ambulatorial Contínua (DPAC) e a Diálise Peritoneal Automatizada (DPA). Segundo o censo brasileiro de 2016 estimava-se que em torno de 123 mil pacientes encontravam-se em tratamento dialítico no Brasil, 92,1\% por hemodiálise e $7,9 \%$ por DP sendo que DPA era a modalidade predominante (SESSO RCC, et al., 2017). No entanto, a principal complicação da DP é o risco de ocorrência de peritonite, com sérias consequências que podem levar à remoção de cateter e óbito (BROWN MC, et al., 2011).

Os micro-organismos mais frequentemente associados à peritonite são bactérias Gram-positivas, principalmente Staphylococcus, e bactérias Gram-negativas (MUJAIS S, 2006; BARRETTI P, et al., 2007; VAN ESCH S, et al., 2014). No Brasil, conforme a região há predominância de S. aureus ou Staphylococcus coagulase-negativos (SCN) (MORAES TP, et al., 2009; LOBO JVD, et al., 2010; OLIVEIRA LG, et al., 2012; FIGUEIREDO AE, et al., 2013; CAMARGO CH, et al., 2014).

O desfecho da peritonite pode ser influenciado pelo tipo de agente etiológico, além do ambiente e do contexto social dos pacientes. A taxa de resolução pode ser menor em infecções por $S$. aureus ou por Gram-negativos, enquanto que a remoção de cateter e mortalidade são mais elevadas em peritonite fúngica (BUNKE CM, et al, 1997; BARRETTI P, et al., 2007; GIACOBINO J, et al., 2016).

Para prevenção e redução da ocorrência de peritonite, recomendações têm sido estabelecidas pela Sociedade Internacional de Diálise Peritoneal (ISPD), incluindo treinamento dos pacientes e seus cuidadores além de acompanhamento por uma equipe multiprofissional (PIRAINO B, et al., 2011; LI PK, et al., 2016). Também é recomendado que a taxa de peritonite não seja superior a 0,5 episódio/paciente/ano (ep./ano) ou preferencialmente inferior a 0,36 ep./ano ou um episódio a cada 33 meses (PIRAINO B, et al., 2011; LI PK, et al., 2016). 
Estudos brasileiros têm relatado a ocorrência de peritonite e suas consequências em diferentes sistemas de diálise peritoneal, porém, há grandes diferenças regionais. No Rio Grande do Sul há publicações anteriores, porém, esse é o primeiro estudo realizado na região norte do estado (JACOBOWSKI JAD, et al., 2005; FIGUEIREDO AE, et al., 2013).

Diante do exposto, o objetivo deste estudo foi avaliar as taxas e os desfechos de peritonite, além de dados epidemiológicos, clínicos e microbiológicos dos casos de peritonite em pacientes submetidos à DPA em um centro de diálise da cidade de Passo Fundo, RS, em um período de 18 anos.

\section{MÉTODOS}

O protocolo de pesquisa foi aprovado pelo Comitê de Ética em Pesquisa (CEP) da Universidade de Passo Fundo, RS, com parecer no.1.769.143.

Neste estudo retrospectivo foi analisado um banco de dados de todos os pacientes submetidos à DPA no Serviço de Nefrologia do Hospital de Clínicas de Passo Fundo (HCPF), RS, Brasil, no período de junho de 1999 a novembro de 2016. Foram incluídos os pacientes com 15 anos ou mais de idade e que desenvolveram pelo menos 1 episódio de peritonite. Foram coletados dados sociodemográficos (idade, sexo, raça, nível educacional, profissão e renda familiar), dados clínicos (causas da doença renal crônica, sintomatologia, número de episódios, data de início e duração de cada episódio de peritonite, desfecho da peritonite) e laboratoriais (micro-organismo causador, contagem de leucócitos no fluido de diálise), sendo assegurado o sigilo desses dados. Pacientes com tempo inferior a 30 dias de DP ou que não possuíam dados completos foram excluídos da análise.

Os critérios para o diagnóstico de peritonite, classificação e desfecho dos episódios (cura, recorrência, recidiva, refratoriedade), cálculo da taxa de peritonite, expressa como episódio/paciente/ano (ep./ano), e orientações terapêuticas foram definidos de acordo com as diretrizes da ISPD (LI PK, et al., 2010; PIRAINO B, et al., 2011; LI PK, et al., 2016). Recidivas de episódios não foram computadas como mais um episódio durante o cálculo das taxas de peritonite e os episódios recorrentes e repetidos foram computados como novo episódio de peritonite. O treinamento do paciente e do cuidador, assim como o acompanhamento dos pacientes, foram executados por um único profissional da enfermagem. Os pacientes eram orientados para aplicar creme de gentamicina ou mupirocina diariamente junto ao local de saída do cateter. O implante do cateter (Swan Neck tipo Missouri) foi realizado sempre pelo mesmo cirurgião. Este protocolo foi mantido durante todos o período analisado.

A cultura e a identificação dos micro-organismos foram realizadas de acordo com os procedimentos de rotina adotados no laboratório do HCPF e recomendações da ISPD (LI PK, et al., 2010; LI PK, et al., 2016).

Para a análise estatística, variáveis categóricas foram descritas como frequência ou percentual e as variáveis contínuas foram expressas como média \pm DP ou mediana. As comparações entre as variáveis contínuas foram feitas pelo teste $t$ de Student. $O$ teste de qui-quadrado foi utilizado para comparações entre as variáveis categóricas. Para a análise dos dados foi utilizado o programa estatístico SPSS versão 20 para Windows (SPSS Inc., Chicago, IL, USA).

\section{RESULTADOS}

Durante o período analisado de 18 anos, 213 pacientes foram submetidos à DPA. A proporção, em média, de pacientes livres de peritonite durante um ano foi de $80,6 \%$. Do total de pacientes, 88 (41,3\%) apresentaram pelo menos 1 episódio de peritonite, somando 181 episódios.

A Tabela 1 mostra as características gerais dos 88 pacientes que apresentaram episódios de peritonite. Houve predominância do sexo feminino $(53,4 \%)$ e da raça branca $(87,5 \%)$, a idade média foi de $51,8 \pm 16,1$ anos e aproximadamente um terço dos pacientes tinha idade superior a 60 anos. A maioria tinha ensino fundamental completo $(78,5 \%), 67 \%$ eram solteiros, $87 \%$ tinham renda salarial até 5 salários mínimos e quase a totalidade era procedente do estado do Rio Grande do Sul. Diabetes foi a causa mais frequente da DRC (36\%) 
Tabela 1 - Características gerais de 88 pacientes submetidos à DPA. Passo Fundo, RS,1999-2016.

\begin{tabular}{|c|c|}
\hline Características & $\mathbf{N}(\%)$ \\
\hline \multicolumn{2}{|l|}{ Sexo } \\
\hline Feminino & $47(52,4)$ \\
\hline Masculino & $41(46,6)$ \\
\hline \multicolumn{2}{|l|}{ Raça } \\
\hline Branco & $77(87,5)$ \\
\hline Pardo & $8(9,1)$ \\
\hline Negro & $3(3,4)$ \\
\hline Idade média \pm DP (anos), & $51,8 \pm 16,1$ \\
\hline Pacientes com idade $\geq 60$ anos & $27(30,7)$ \\
\hline \multicolumn{2}{|l|}{ Nível educacional } \\
\hline Ensino fundamental & $69(78,5)$ \\
\hline Ensino médio & $9(10,2)$ \\
\hline Ensino superior & $6(6,8)$ \\
\hline Analfabeto & $4(4,5)$ \\
\hline \multicolumn{2}{|l|}{ Profissão } \\
\hline Do lar & $34(38,6)$ \\
\hline Agricultor & $17(19,3)$ \\
\hline Aposentado & $13(14,8)$ \\
\hline Estudante & $4(4,5)$ \\
\hline Outras & $32(22,8)$ \\
\hline \multicolumn{2}{|l|}{ Renda familiar } \\
\hline De até 1 salário mínimo & $27(30,7)$ \\
\hline De 2 à 5 salários mínimos & $50(56,8)$ \\
\hline Mais de 5 salários mínimos & $11(12,5)$ \\
\hline \multicolumn{2}{|l|}{ Distância do centro de diálise (Km) } \\
\hline Até 100 & $61(70,0)$ \\
\hline \multicolumn{2}{|l|}{ Estado civil } \\
\hline Solteiro & $59(67,0)$ \\
\hline Casado & $25(28,4)$ \\
\hline Viúvo & $4(4,6)$ \\
\hline \multicolumn{2}{|l|}{ Procedência do paciente } \\
\hline Rio Grande do Sul & $57(98,9)$ \\
\hline \multicolumn{2}{|l|}{ Causa de DRC } \\
\hline Nefropatia diabética & $32(36,4)$ \\
\hline Insuficiência renal crônica não específica & $24(27,3)$ \\
\hline Rim policístico autossômico dominante & $11(12,5)$ \\
\hline Doença renal hipertensiva & $12(13,6)$ \\
\hline Outros & $9(10)$ \\
\hline
\end{tabular}

Fonte: Serviço de Nefrologia do HCPF, 2017.

Em 181 episódios de peritonite, dor abdominal (86,7\%), efluente de diálise turvo $(80,7 \%)$ e febre (32\%) foram os mais frequentes. Contagem de leucócitos no fluido peritoneal acima de $100 / \mu \mathrm{L}$ com predominância de polimorfonucleares ( $>50 \%$ ) ocorreu em mais de $95 \%$ dos casos, sendo que as medianas foram de $2.076 / \mu \mathrm{L}$ nas peritonites causadas por Gram-positivos e de 5.200/ $\mathrm{L}$ por Gram-negativos. 
A Tabela 2 mostra a frequência dos casos de peritonite em função do agente etiológico identificado na cultura. Não houve detecção de cepas de $S$. aureus resistentes à meticilina (MRSA). Todos os casos de Enterococcus foram sensíveis à oxacilina e não houve casos de Enterococcus resistentes à vancomicina (VRE).

Tabela 2 - Distribuição dos micro-organismos causadores de episódios de peritonite. Passo Fundo, RS,1999-2016. $(n=177)$

\section{Micro-organismo causador}

\section{Gram-positivos}

Staphylococcus coagulase-negativo (SCN)

Staphylococcus aureus

Bacillus spp

Streptococcus spp

Enterococcus spp

Outros
No. Episódios (\%)

$68(37,5)$

$26(14,3)$

$23(12,7)$

$5(2,8)$

$7(3,8)$

$5(2,8)$

$2(1,1)$

\section{Gram-negativos}

$29(16,0)$

\section{Escherichia coli}

Enterobacter spp

Acinetobacter spp

Klebsiella spp

Outros

\section{Fungos}

\begin{tabular}{lc}
\hline Candida spp & $11(6,1)$ \\
\hline Polimicrobiano & $11(6,1)$ \\
\hline Cultura negativa & $58(32,0)$ \\
\hline Não registrado & $4(2,3)$ \\
\hline
\end{tabular}

Fonte: Serviço de Nefrologia do HCPF, 2017.

Quando foram comparados dois períodos de tempo, de 1998 a 2007 e de 2008 a 2016, embora não tenha havido significância estatística, houve um declínio na proporção de casos de $S$. aureus (de 15,4\% para $5,3 \%$ ) e de cultura mista (8,1\% para 1,7\%), com aumento de SCN (de 13,8\% para 15,5\%) e de bactérias Gram-negativas (de 13,8\% para 18,9\%) ( $p=0,063$ ). Na Tabela 3 são apresentados os dados relativos aos episódios de peritonite. A taxa total de peritonite foi de $0,33 \mathrm{ep}$./ano, para SCN foi de 0,048 , para $S$. aureus de 0,042 e para bactérias Gram-negativas de 0,057. 
Tabela 3 - Características relacionadas aos episódios de peritonite $(n=181)$.

\begin{tabular}{|c|c|}
\hline Características & $\mathbf{N}$ \\
\hline Taxa total de peritonite (ep./ano) & 0,33 \\
\hline Taxa de peritonite para SCN (ep./ano & 0,048 \\
\hline Taxa de peritonite para $S$. aureus (ep./ano) & 0,042 \\
\hline Taxa de peritonite para Gram-negativos (ep./ano) & 0,057 \\
\hline No. episódios de peritonite por paciente, média \pm DP & $2,1 \pm 1,4$ \\
\hline \multicolumn{2}{|l|}{ No. de pacientes com episódios de peritonite, $n .^{\circ}(\%)$} \\
\hline Somente 1 episódio & $42(47,7)$ \\
\hline até 2 & $23(26,1)$ \\
\hline até 3 & $10(11,4)$ \\
\hline 4 a 8 & $13(15,0)$ \\
\hline \multicolumn{2}{|l|}{ Classificação do episódio, n. pacientes (\%) } \\
\hline Primeiro episódio & $88(48,6)$ \\
\hline Novo episódio & $80(44,2)$ \\
\hline Recidiva & $9(5,0)$ \\
\hline Recorrente & $4,(2,2)$ \\
\hline \multicolumn{2}{|l|}{ Troca da bolsa de diálise, n.ำ pacientes (\%) } \\
\hline Paciente & $38(43,2)$ \\
\hline Esposo (a) & $18(20,4)$ \\
\hline Outro familiar & $31(35,3)$ \\
\hline Profissional contratado & $1(1,1)$ \\
\hline Dias de internação, média \pm DP & $11,9 \pm 7,4$ \\
\hline $\begin{array}{l}\text { Tempo do início em DPA e o primeiro episódio, mediana } \\
\text { (meses) }\end{array}$ & $29(0-149)$ \\
\hline
\end{tabular}

Fonte: Serviço de Nefrologia do HCPF, 2017.

Em relação ao desfecho, no total de casos houve cura em $74 \%$ e óbito em $6,6 \%$. Quando os microorganismos foram analisados conjuntamente e comparados para verificar uma possível associação com as variáveis cura, remoção de cateter e óbito, houve diferença estatisticamente significativa no desfecho (Quiquadrado $p<001$ ), conforme pode ser observado na Tabela 4. 
Tabela 4 - Desfecho dos episódios de peritonite em 88 pacientes submetidos à DPA em relação aos microorganismos causadores. Passo Fundo, RS,1999-2016. $(n=177)$

\begin{tabular}{c|c|c|c|c}
\hline Micro-organismo & Cura & $\begin{array}{c}\text { Remoção } \\
\text { cateter }\end{array}$ & Óbito & Total \\
\hline Gram-positivo & $55(82,1 \%)$ & $8(11,9 \%)$ & $4(6,0 \%)$ & $67(100 \%)$ \\
Gram-negativo & $20(60,6 \%)$ & $8(24,2 \%)$ & $5(15,2 \%)$ & $33(100 \%)$ \\
Misto & $4(50,0 \%)$ & $3(37,5 \%)$ & $1(12,5 \%)$ & $8(100 \%)$ \\
Fungo & $2(18,2 \%)$ & $8(72,7 \%)$ & $1(9,1 \%)$ & $11(100 \%)$ \\
Cultura negativa & $50(86,2 \%)$ & $7(12,1 \%)$ & $1(1,7 \%)$ & $58(100 \%)$ \\
Não registrado & 4 & - & - & - \\
Total & $135(74,6 \%)$ & $34(18,8 \%)$ & $12(6,6 \%)$ & $181(100 \%)$ \\
\hline
\end{tabular}

Teste Qui-quadrado: $p<0,001$

Fonte: Serviço de Nefrologia do HCPF, 2017.

\section{DISCUSSÃO}

Os resultados deste estudo confirmam que peritonite continua sendo uma complicação importante em pacientes submetidos à DP. A proporção, em média, de pacientes livres de peritonite durante um ano foi de $80,6 \%$, valor que está dentro do limite aceitável recomendado pela ISPD (PIRAINO B, et al., 2011). Quase a metade $(41,3 \%)$ dos pacientes desenvolveu peritonite, num total de 181 episódios. A ocorrência de peritonite em pacientes submetidos à DP varia conforme o local, o número de pacientes e o período analisado, situando-se entre $42 \%$ e $88 \%$ em alguns relatos brasileiros (MORAES TP, et al., 2009; LOBO JVD, et al., 2010; FERREIRA JJ, et al., 2011).

Quanto às características sociodemográficas (Tabela 1), houve ligeira predominância do sexo feminino, a idade média foi de 52 anos e um terço dos pacientes tinha idade superior a 60 anos. Esses e outros dados são similares ou há pequenas diferenças em relação aos verificados em outros estudos brasileiros (MORAES TP, et al., 2009; LOBO JVD, et al., 2010; FIGUEIREDO AE, et al., 2013; SESSO RCC, et al., 2017).

$\mathrm{Na}$ literatura internacional são encontrados resultados bastante variáveis como, por exemplo, na Argentina onde 18\% eram idosos enquanto nos EUA e Canadá representaram $42 \%$ e $52 \%$, respectivamente (MUJAIS S, 2006; LOBO J, et al., 2011). Segundo Pecoits-Filho R, et al. (2017) as condições socioeconômicas e geográficas podem ter impacto sobre a utilização de DPA. Cerca de $78 \%$ de nossos pacientes tinham o ensino fundamental e somente $4,5 \%$ eram analfabetos, com a maioria tendo uma renda entre 2 a 5 salários mínimos. Esses valores refletem melhores condições comparadas às de outros estudos brasileiros e podem ter contribuído para manter as taxas de peritonite em limites aceitáveis, uma vez que a baixa escolaridade pode ser um fator de risco para o desenvolvimento de peritonite (FERNANDES N, et al., 2008; LOBO JVD, et al., 2010).

Em $36 \%$ dos pacientes avaliados neste estudo, a causa da DRC foi nefropatia diabética. Este valor é próximo ao relatado por outros autores (MUJAIS S, 2006; LOBO JVD, et al., 2010; KOFTERIDIS DP, et al., 2010; OLIVEIRA LG, et al., 2012). Porém, foi superior aos verificados na Argentina, na Holanda e no sul do Brasil (LOBO J, et al., 2011; FIGUEIREDO AE, et al., 2013; VAN ESCH S, et al., 2014).

De acordo com o Censo Brasileiro de Diálise de 2014, o percentual de pacientes diabéticos foi de $29 \%$ (SESSO RCC, et al., 2017). Dos achados clínicos, dor abdominal e efluente turvo foram os mais frequentes em mais de $80 \%$ dos pacientes. Esses achados também foram os de maior frequência em outros estudos (OLIVEIRA LG, et al., 2012; BARRETTI P, et al., 2012; CAMARGO CH, et al., 2014). Esses achados clínicos são resultantes da presença e da replicação de micro-organismos, principalmente bactérias, na cavidade 
abdominal e que induzem uma resposta inflamatória com afluxo de leucócitos polimorfonucleares e liberação de substâncias inflamatórias responsáveis pela dor. Embora outras causas possam ser responsáveis, esses são critérios presuntivos para a suspeita de peritonite (LI PK, et al., 2010).

Em $60 \%$ dos episódios houve isolamento de apenas 1 micro-organismo, com frequência maior de bactérias Gram-positivas, predominando SCN e S. aureus (Tabela 2). Barretti P, et al. (2007) relataram que no Brasil a maioria dos casos de peritonite é causada por SCN e $S$. aureus, com grandes diferenças regionais. Alguns estudos brasileiros referem predominância de SCN ou de $S$. aureus (MORAES TP, et al., 2009; LOBO JVD, et al., 2010; OLIVEIRA LG, et al., 2012; FIGUEIREDO AE, et al., 2013; CAMARGO CH, et al., 2014). Já Pecoits-Filho R, et al. (1998) relataram que no período de 1980 a 1995, S. aureus foi o agente etiológico mais frequente enquanto que, de 1999 a 2003, S. epidermidis foi o mais prevalente Relatos internacionais também citam micro-organismos Gram-positivos, com predominância de SCN (KOFTERIDIS DP, et al., 2010; VAN ESCH S, et al., 2014).

A predominância de Staphylococcus como causa de peritonite pode estar associada à presença desses micro-organismos na microbiota da pele, garganta e mucosa nasal, podendo ser introduzidos no momento da conexão do sistema (SESSO R, et al., 1989). A aplicação diária de mupirocina no sítio de saída do cateter pode reduzir infecções por S. aureus (VAN ESCH S, et al., 2014). Esta observação também foi feita por Barretti P, et al. $(2007,2012)$ pelo uso de mupirocina e tratamento de carreadores crônicos de $S$. aureus, com aumento de casos por bacilos gram-negativos com o passar dos anos. Quando analisamos dois períodos de 8 anos, embora não estatisticamente significativo $(p=0,063)$, houve declínio de um período para outro na proporção de casos de $S$. aureus, com aumento na frequência de SCN e de bactérias Gramnegativas. Essas oscilações, no entanto, não tiveram influência no desfecho e possivelmente devem ter ocorrido devido à diferença no número de pacientes nos dois períodos.

Gram-negativos responderam por $16 \%$ dos episódios, com predominância de E. coli, valor inferior aos verificados na América do Norte e diferentes regiões do Brasil (MUJAIS S, 2006; MORAES TP, et al., 2009; FIGUEIREDO AE, et al., 2013). Foi, no entanto, superior aos encontrados em São Paulo e na Holanda (BEVILACQUA JL, et al., 1995; VAN ESCH S, et al., 2014). Os resultados deste estudo em relação à peritonite fúngica $(6,2 \%)$ são semelhantes ou pouco superiores aos descritos por outros pesquisadores (MUJAIS S, 2006; KOFTERIDIS DP, et al., 2010; VAN ESCH S, et al., 2014).

Cultura negativa ocorreu em $32,7 \%$ dos episódios de peritonite. Valores encontrados em diferentes regiões do Brasil podem variar de 16,9\% a 41,2\% (LOBO JVD, et al., 2010; PERES LAB, et al., 2011; FIGUEIREDO AE, et al., 2013). Maujais S (2006) compara os valores de cultura negativa relatados por diferentes autores em diversos países, com variação de $9,4 \%$ a 36,9\%. Na Austrália foi encontrado um valor de $12 \%$ (FAHIM M, et al., 2010). A ISPD recomenda valores abaixo de $20 \%$ de culturas negativas (LI PK, et al., 2010) e sugere que os procedimentos de amostragem e cultura devem ser revisados quando o valor exceder $15 \%$ (LI PK, et al., 2016). Neste estudo possivelmente houve influência da metodologia utilizada pelo laboratório, que foi sofrendo modificações com o passar dos anos. Outras possibilidades seriam a baixa carga de micro-organismos em alguns pacientes, exposição recente do paciente a antibióticos e eventual presença de micro-organismos incomuns ou intracelulares (FAHIM M, et al., 2010; LI PK, et al., 2016).

Em quase $47,7 \%$ de nossos pacientes houve somente 1 episódio de peritonite e a mediana para a ocorrência do primeiro episódio foi de 28 meses. Peritonite refratária ocorreu em $61,7 \%$ dos episódios. Segundo as diretrizes da ISPD, recomenda-se como meta valor não superior a um episódio a cada 18 meses (LI PK, et al., 2010). Um aspecto positivo de nosso estudo foi a taxa de peritonite de 0,33 ep./ano, abaixo do que é sugerido pela ISPD, que é de 0,36 ep./ano (PIRAIANO B, et al., 2011).

Um fator que pode ter contribuído para manter esta taxa aceitável foi a utilização de DPA para todos os pacientes desde o início do tratamento dialítico. Este tipo de procedimento diminui a possibilidade de contaminação do paciente relativa ao processo de conexão/desconexão do sistema (SZETO C, et al., 2014). Estudos nacionais mostraram taxas divergentes em diferentes regiões do país (PECOITS-FILHO RFS, et al., 1998; LOBO JVD, et al., 2010; PERES LAB, et al., 2011; BARRETTI P, et al., 2012). 
Moraes TP et al. (2009) verificaram uma incidência de 0,82 ep./ano, com as taxas diminuindo com o passar dos anos, principalmente com a introdução do sistema double-bag. Oliveira LG et al. (2012) relataram 0,65 ep./ano, valor próximo ao verificado por Figueiredo AE et al. (2013), que foi de 0,63 ep/ano com mediana de 0,41 . Em um estudo multicêntrico nacional, Fernandes $N$ et al. (2008) reportaram uma incidência de 0,4 ep./ano. Em outros países também existe divergência em relação à incidência de peritonite (FAHIM M, et al., 2010; LOBO J, et al., 2011). Nos EUA e no Canadá as taxas encontradas foram 0,36 ep./ano e 0,43 ep./ano, respectivamente (MAUJAIS S, 2006).

As taxas de peritonite por micro-organismo foram de 0,048 para SCN, 0,042 para $S$. aureus e 0,057 para bactérias Gram-negativas (Tabela 3). A taxa para $S$. aureus foi inferior ao limite recomendado pela ISPD, que é de 0,06 (PIRAINO B, et al., 2011). Um estudo indiano reportou taxas de 0,11 para Gram-positivos e 0,17 para Gram-negativos, onde somente $61,3 \%$ das culturas foram positivas (PRASAD N, et al., 2003). Figueiredo $A E$ et al. (2013) relataram uma incidência de 0,18 ep./ano para SCN e 0,12 ep./ano para S. aureus e germes Gram-negativos em geral. No estudo de Barretti $P$ et al. (2012) foi verificada uma redução significativa na incidência de peritonite por $S$. aureus de 0,13 para 0,04 ep./ano quando analisados dois períodos de tempo. Camargo $\mathrm{CH}$ et al. (2014) analisaram 115 episódios de peritonite por SCN entre os anos de 1994 e 2011 e verificaram uma incidência de 0,15 ep./paciente/ano, que não variou significativamente quando três períodos foram avaliados. Quando analisamos dois períodos de 8 anos, embora não estatisticamente significativo, houve discreta diminuição na taxa de peritonite para $S$. aureus com pequeno aumento para SCN e bactérias Gram-negativas.

Quanto aos desfechos dos episódios de peritonite, em 74\% do total de casos houve cura, comparável aos valores observados por alguns autores (BEVILACQUA JL, et al., 1995; PERES LAB, et al., 2011). Foi inferior aos relatados por outros (PIRAINO B, et al., 2011; FERREIRA JJ, et al., 2011; PECOITS-FILHO R, et al., 2017). Neste estudo houve uma piora significativa no desfecho das infecções por Gram-negativos e fungos, com taxas de cura de 60,6\% e 18,8\%, respectivamente (Tabela 4). Entre os Gram-positivos, resolução ocorreu em $84,6 \%$ dos episódios causados por SCN e em $73,9 \%$ por $S$. aureus. Na América Latina a taxa de resolução varia de $55 \%$ a $78 \%$, sendo pior nos episódios causados por $S$. aureus quando comparado aos causados por SCN (BARRETTI P, et al., 2007). Já Oliveira LG et al. (2012) não observaram diferença significativa em relação a essas duas espécies de estafilococos. As taxas de remoção de cateter e óbito foram significativamente maiores em infecções por Gram-negativos e fungos do que por Grampositivos (Tabela 4). Fontan $P$ et al. (2005) também encontraram uma taxa maior de mortalidade por fungos, comparada à causada por Gram-negativos e $S$. aureus. Bunke CM et al. (1997) relataram valores superiores a $80 \%$ de cura para estafilococos e $63 \%$ para Gram-negativos não Pseudomonas, com remoção de cateter em $30 \%$ dos episódios causados por Gram-negativos. Ao compararmos os dados em dois períodos de 8 anos não encontramos diferença significativa em relação aos desfechos, ao contrário do estudo de Oliveira LG et al. (2012) onde os desfechos foram diferentes em dois períodos analisados. No estudo de Giacobino $\mathrm{J}$ et al. (2016) cerca de $7 \%$ dos pacientes desenvolveram peritonite fúngica, principalmente por Candida, sendo que em todos os casos houve remoção do cateter e $26 \%$ evoluíram a óbito.

Embora a alta prevalência de culturas negativas (32,7\%), bem acima do limite do recomendado pela ISPD, aparentemente não houve influência nos desfechos dos episódios. Resolução (85\%) e remoção de cateter $(12,1 \%)$ tiveram valores muito próximos aos de infecções por Gram-positivos. Chama a atenção que somente em $1,7 \%$ desses casos ( 1 em 58 ) ocorreu óbito (Tabela 4). Um valor superior a $85 \%$ de cura foi verificado por Fernandes $\mathrm{N}$ et al. (2008) e Moraes TP et al. (2009), apesar de culturas negativas em $40 \%$ e $41,5 \%$ dos casos, respectivamente. Já Lobo JVD et al. (2010) observaram $32,5 \%$ de culturas negativas com $77,8 \%$ de cura.

O presente estudo apresenta limitações. Trata-se de uma análise retrospectiva e foi realizada a partir de dados de um único centro. Não foram incluídos dados sobre a contagem de leucócitos no acompanhamento da peritonite e sobre o perfil de sensibilidade bacteriana aos antimicrobianos. Também não foi considerada uma possível influência de outros fatores, como por exemplo, estado nutricional. Portanto, se deve observar as particularidades para não generalizar os resultados. Outra limitação foi a ocorrência de um número elevado de culturas negativas, superior às recomendações da ISPD. Nesse sentido, é necessário um 
aprimoramento dos procedimentos de amostragem e cultura para reduzir a frequência de resultados negativos, medidas que já foram adotadas no serviço. Futuros estudos deverão incluir dados sobre o comportamento dos micro-organismos frente aos antimicrobianos assim como avaliar possíveis fatores de risco no desenvolvimento de peritonite em pacientes submetidos à DP.

\section{CONCLUSÃO}

De uma maneira geral, embora com limitações, este estudo revelou que as características gerais são similares às de estudos anteriores. Porém, os desfechos foram influenciados pelo micro-organismo causador, sendo mais desfavoráveis nos episódios causados por bactérias Gram-negativas e fungos. Entre os pontos fortes do estudo, pode-se destacar que o procedimento de diálise (DPA) foi o mesmo para todos os pacientes desde o início, o treinamento do paciente/cuidador foi efetuado sempre pela mesma enfermeira e, decorrentes disto, as taxas de peritonite se mantiveram dentro dos limites preconizados pela ISPD durante todo o período analisado. Tais fatos reforçam a importância da manutenção e do monitoramento constante dos procedimentos.

\section{AGRADECIMENTOS}

À enfermeira Tania Eleonor Baldo pelo treinamento dos cuidadores, assim como o acompanhamento dos pacientes. À Dra. Ivana Loraine Lindemann pelo auxílio na análise dos dados.

\section{REFERÊNCIAS}

1. BARRETTI P, et al. Peritoneal Dialysis-Related Peritonitis Due to Staphylococcus aureus: A Single-Center Experience over 15 Years. PLoS One, 2012; 7(2):e31780.

2. BARRETTI P, et al. Peritonitis in Latin America. Perit Dial Int, 2007; 27(3):332-39.

3. BEVILACQUA JL, et al. Diálise peritoneal ambulatorial contínua (DPAC): experiência de 10 anos em um centro brasileiro. J Bras Nefrol, 1995; 17(4):206-13.

4. BROWN MC, et al. Scottish Renal Registry. Peritoneal dialysis-associated peritonitis rates and outcomes in a national cohort are not improving in the post-millennium (2000-2007). Perit Dial Int, 2011; 31(6):639-50.

5. BUNKE CM, et al. Outcomes of single-organism peritonitis in peritoneal dialysis: Gram-negatives versus Grampositives in the Network 9 peritonitis study. Kidney Int, 1997; 52:524-9.

6. CAMARGO $\mathrm{CH}$, et al. Peritoneal Dialysis-Related Peritonitis due to Coagulase-Negative Staphylococcus: A Review of 115 Cases in a Brazilian Center. Clin J Am Soc Nephrol, 2014; 9:1074-81.

7. DURVASULA RV, HIMMERFALB J. Chronic renal failure and dialysis. ACP Med, 2011; 1-17.

8. FAHIM M, et al. Culture-Negative Peritonitis in Peritoneal Dialysis Patients in Australia: Predictors, Treatment, and Outcomes in 435 Cases. Am J Kidney Dis, 2010; 55:690-7.

9. FERNANDES N, et al. The Brazilian Peritoneal Dialysis Multicenter Study (BRAZPD): characterization of the cohort. Kidney Int, 2008; 73:145-51.

10. FERREIRA JJ, et al. Manifestação clínica de peritonite em pacientes que vivem com insuficiência renal crônica. Arq Bras Cienc Saúde, 2011; 36(3):150-4.

11. FIGUEIREDO AE, et al. Peritonites em pacientes em diálise peritoneal: análise de um centro brasileiro segundo as recomendações da Sociedade Internacional de Diálise Peritoneal. J Bras Nefrol, 2013; 35(3):214-219.

12. FONTAN $P$, et al. Peritonitis-related mortality in patients undergoing chronic peritoneal dialysis. Perit Dial Int, 2005; 25(3):274-84.

13. GIACOBINO J, et al. Fungal peritonitis in patients undergoing peritoneal dialysis (PD) in Brazil: molecular identification, biofilm production and antifungal susceptibility of the agents. Med Mycol, 2016; 54(7):725-32.

14. JACOBOWSKI JAD, et al. Pacientes com Insuficiência Renal Crônica: Causas de saída do Programa de Diálise Peritoneal. Rev Gaúcha Enferm, 2005; 26(3):381-91.

15. KOFTERIDIS DP, et al. Peritoneal dialysis-associated peritonitis: clinical features and predictors of outcome. Int J Infec Dis, 2010; 14: e489-e93.

16. LI PK, et al. International Society for Peritoneal Dialysis. Peritoneal dialysis-related infections recommendations: 2010 update. Perit Dial Int, 2010; 30(4):393-423.

17. LI PK, et al. ISPD peritonitis recommendations: 2016 update on prevention and treatment. Perit Dial Int, 2016; 36(5):481-508.

18. LOBO J, et al. Peritoneal dialysis in Argentina. A nationwide study. Perit Dial Int, 2011; 31(1):19-26. 
19. LOBO JVD, et al. Preditores de peritonite em pacientes em um programa de diálise peritoneal. J Bras Nefrol, 2010; 32(2):156-64.

20. MORAES TP, et al. Peritoneal dialysis in Brazil: twenty-five years of experience in a single center. Perit Dial Int, 2009; 29(5):492-98.

21. MUJAIS S. Microbiology and outcomes of peritonitis in North America. Kidney Int, 2006; 70: S55-S62.

22. OLIVEIRA LG, et al. Peritonitis in recent years: clinical findings and predictors of treatment response of 170 episodes at a single Brazilian center. Int Urol Nephrol, 2012; 44:1529-1537.

23. PECOITS-FILHO R, et al. Racial and social disparities in the access to automated peritoneal dialysis - results of a national PD cohort. Sci Rep, 2017; 7(1):5214-21.

24. PECOITS-FILHO RFS, et al. Diálise peritoneal contínua ambulatorial (DPCA): experiência de 15 anos em Curitiba. J Bras Nefrol, 1998; 20(1):22-30.

25. PERES LAB, et al. Peritonites em diálise peritoneal contínua. Rev Bras Clin Med, 2011; 9(5):350-3.

26. PICCOLLI AP, et al., Prevalência da doença renal crônica em uma população do Sul do Brasil (estudo ProRenal). Braz. J. Nephrol. (J. Bras. Nefrol.), 2017; 39(4):384-390.

27. PIRAINO B, et al. ISPD position statement on reducing the risks of peritoneal dialysis-related infections. Perit Dial Int, 2011; 31(6):614-630.

28. PRASAD N, et al. Outcome of gram-positive and gram-negative peritonitis in patients on continuous ambulatory peritoneal dialysis: a single-center experience. Perit Dial Int, 2003; 23(Supl. 2):144-147.

29. SESSO R, et al. Staphylococcus aureus skin carriage and development of peritonitis in patients on continuous ambulatory peritoneal dialysis. Clin Nephrol, 1989; 31:264-8.

30. SESSO RCC, et al. Inquérito Brasileiro de Diálise Crônica. J Bras Nefrol, 2017; 39(3):261-66.

31. SZETO C. Peritonitis rates of the past thirty years: from improvement to stagnation. Perit Dial Int, 2014; 34(2):151-153.

32. VAN ESCH S, et al. 32 years' experience of peritoneal dialysis-related peritonitis in a university hospital. Perit Dial Int, 2014; 34(2):162-70. 